\title{
BMJ Open Community social support and onset of dementia in older Japanese individuals: a multilevel analysis using the JAGES cohort data
}

\author{
Yasuhiro Miyaguni (D) ,,2 Takahiro Tabuchi (D) , 3 Jun Aida (D) ,4 \\ Masashige Saito (D) , ${ }^{1,5}$ Taishi Tsuji (D) , ${ }^{6}$ Yuri Sasaki, ${ }^{7}$ Katsunori Kondo (D) ${ }^{2,8}$
}

To cite: Miyaguni Y, Tabuchi T, Aida J, et al. Community social support and onset of dementia in older Japanese individuals: a multilevel analysis using the JAGES cohort data. BMJ Open 2021;11:e044631. doi:10.1136/ bmjopen-2020-044631

- Prepublication history and supplemental material for this paper is available online. To view these files, please visit the journal online (http://dx.doi. org/10.1136/bmjopen-2020044631).

Received 08 September 2020 Accepted 20 May 2021

Check for updates

(C) Author(s) (or their employer(s)) 2021. Re-use permitted under CC BY-NC. No commercial re-use. See rights and permissions. Published by BMJ.

For numbered affiliations see end of article.

Correspondence to Dr Yasuhiro Miyaguni; miyaguni@n-fukushi.ac.jp

\section{ABSTRACT}

Objective Recently, there has been an increase in the number of people with dementia. However, no study has examined the association between community-level social support and the onset of incident dementia using multilevel survival analysis.

Design A prospective cohort study.

Participants and setting We analysed data pertaining to 15313 (7381 men and 7932 women) community-dwelling adults aged 65 years or older who had not accessed longterm care insurance and were living in Aichi Prefecture (seven municipalities) in Japan.

Primary and secondary outcome measures The association between community-level social support and onset of incident dementia was examined using the Japan Gerontological Evaluation Study, a prospective cohort study introduced in Japan in 2003. Incident dementia was assessed using Long-term Care Insurance records spanning 3436 days from the baseline survey.

Results During the 10-year follow-up, the onset of incident dementia occurred in 1776 adults. Among older people, a 1\% increase in community-level social support (in the form of receiving emotional support) was associated with an approximately $4 \%$ reduction in the risk of developing dementia, regardless of socio-demographic variables and health conditions $(\mathrm{HR}=0.96 ; 95 \% \mathrm{Cl}=0.94$ to 0.99 ).

Conclusions Receiving community-level social support in the form of emotional support is associated with a lower risk of developing incident dementia.

\section{INTRODUCTION}

Dementia constitutes a pressing health challenge, especially among the older population. The incidence of dementia worldwide is projected to rise to 66 and 131 million by 2030 and 2050, respectively. ${ }^{1}$ In Japan alone, it is predicted that there will be 4.62 and 7 million people affected by 2012 and 2025, respectively. These rates suggest that about one in seven Japanese people aged 65 years or above may develop dementia. ${ }^{2}$

Currently, no effective therapeutic intervention for dementia has been determined. As

\section{Strengths and limitations of this study}

- To date, no study has examined the association between community-level social support and the onset of incident dementia using multilevel survival analysis.

- This is a long-term follow-up study that followed older adults in Japan for about 10 years.

- The sample does not fully reflect the older population in Japan because the study subjects were recruited from a single prefecture.

such, identifying adjustable risks and preventive measures is essential for slowing down or preventing the onset of dementia. ${ }^{3}$ Previous studies have identified genetic, vascular and lifestyle-related factors, ${ }^{4-9}$ such as advanced age, being female, having a low education level, being in poor health, smoking and heavy drinking, as being associated with a higher risk of developing dementia. An additional significantly adjustable risk factor is the lack of positive social networks and influences. A previous study suggested that engagement in social activities, and having a rich network of activities within close relationships, confers some protection against dementia among older people. ${ }^{4}$

The definition and the attendant use of the notion of social relationships vary among researchers. The concept may encompass factors such as social participation, social networks and social support. Social support can be defined as aid and assistance exchanged through social relationships and interpersonal transactions, ${ }^{10}$ and it might be a significant protective factor for cognitive ageing. ${ }^{11}$ A previous systematic review paper indicated that people with social support had $50 \%$ lower mortality than those without it. ${ }^{12}$ Social support has been categorised into four types-giving and receiving support 
at an emotional level and at an instrumental level ${ }^{13}-$ all of which occur at an individual level and have been associated with improved health. For example, providing emotional and instrumental social support to nonfamily members leads to fewer depressive symptoms. ${ }^{13}$ Providing emotional support to the spouse, and instrumental support to relatives, friends and neighbours, further lowers the risk of mortality. ${ }^{14}$ Receiving emotional support is associated with improved cognitive function. ${ }^{11}$ In addition, diverse social relationships, including social support from family members, are associated with a lower incidence of dementia. ${ }^{15}$

Besides individual-level social factors, social networks and relationships at the community level have been investigated to understand its association with moderating the risk of functional disability. Such studies investigate 'social capital,' which has been defined as the 'resources that are accessed by individuals as a result of their membership of a network or a group. ${ }^{16}$ A previous study found that lower social capital at the community-level is associated with an increased rate of functional disability among older women. ${ }^{17}$ Moreover, there are reports of research on community social capital and cognitive decline. ${ }^{18}$ However, because these studies are cross-sectional ones, longitudinal studies are needed. Nevertheless, no study has examined how community-level social support influences the risk of dementia. Therefore, this study seeks to evaluate the relationship between social support at the community level and the onset of dementia.

\section{METHODS}

\section{Sample}

Data for this study were accessed via the Japan Gerontological Evaluation Study (JAGES). Set up in Aichi in 2003, this was a prospective cohort study of the Center for Wellbeing and Society of the Nihon Fukushi University. ${ }^{19}$ The research was carried out in seven-three larger (Handa, Tokoname and Chita Hokubu) and four smaller (Agui, Mihama, Minamichita and Taketoyo)-municipalities that encompass the entire southern region of the Chita peninsula and the Aichi Prefecture. In October 2003, an estimated 276208 people resided in these locations where $18.0 \%$ were aged 65 years or above. ${ }^{17}$ On average, the data of 6300 residents in the 44 school districts were analysed in this study. From the three larger municipalities, 5000 survey candidates were randomly selected from the list of persons insured by long-term care insurance who were not certified as requiring long-term care. In the four smaller municipalities, candidates were randomly selected from those not receiving public long-term care insurance benefits due to a physical or cognitive disability. Of the 33152 people selected, 15313 individuals answered the baseline survey (response rate $=52.1 \%$ ). ${ }^{15}$ The exclusion criteria were: (1) people who had difficulty in performing activities of daily living as a result of disabilities; (2) people who did not provide baseline information $(n=579)$; (3) people who did not provide social support information
( $\mathrm{n}=1359)$; and (4) people who did not provide a school district code $(n=2343)$. Ultimately, 11032 subjects (5627 women and 5405 men) were included in this analysis.

\section{Follow-up}

The evaluation parameters of the JAGES Project included health status, functional deterioration and mental impairment among older Japanese people who were not institutionalised. In Japan, there is a long-term care insurance system that covers both institutional and community-based caregiving. Individuals aged 65 years or above qualify to receive benefits on the strict basis of physical and cognitive disability. The follow-up began on 1 November 2003. Dementia-associated data from the six municipalities (specifically in terms of the onset) were assessed until 28 March 2013.

\section{Outcome variables}

Dementia was graded on a scale that includes categories from I to IV and Medical (M based on the Activities of Daily Living Independence Assessment Criteria for Older Individuals with Dementia. The Degree of Autonomy in the Daily Lives of Older Individuals with Dementia Scale, created by the Ministry of Health, Labor, and Welfare of Japan, evaluates an individual's ability to carry out daily tasks associated with living on a scale that includes categories from I to IV and M (online supplemental table 1).

This scale was validated based on its high association with the Mini-Mental State Examination. ${ }^{20}$ It has been reported that dementia symptoms indices are strongly correlated with Mini-Mental State Examination. (Spearman's rank correlation $\rho=-0.73, p<0.001)$. Scores I, II, III and IV on the dementia scale are equivalent to 22,16 , 13 and 6 points on the Mini-Mental State Examination, respectively. ${ }^{20} \mathrm{~A}$ score of I indicates that the patient suffers from some level of cognitive decline but remains able to perform domestic and social tasks nearly independently. A score of II indicates that the patient has certain symptoms or behaviours indicative of cognitive impairment and challenges in communication that may hamper the performance of daily tasks, although some amount of external assistance is needed to facilitate routine function. A score of III indicates that the patient periodically exhibits symptoms indicating communication challenges or symptoms/behaviours, which may interfere with the performance of daily tasks, necessitating external assistance. A score of IV indicates that the patient usually shows communication or behavioural challenges, which hampers performing daily tasks, necessitating frequent care. Finally, a score of M (M=Medical, requires specialised medical care) is used when the patient shows significant cognitive impairment, displays difficult behaviour or has a serious physical illness, requiring expert medical intervention. Symptoms and behaviours seen in the M rank include delirium, paranoia, agitation, self-injury and harm and other psychiatric symptoms, as well as ongoing problem behaviours caused by psychiatric symptoms. 


\section{Explanatory variables}

An aggregate of individual-level background data were acquired for the 44 school-based districts to evaluate community social support. An aggregate of responses for individual-level social support among the school districts was used as an indicator of community social support. Within the Japanese context, school districts (or primary schools) are primary residential units of individuals within rural zones. Generally, school districts comprise geographical settings where older individuals may readily travel via foot or bicycle. ${ }^{21}$

Individual-level social support was assessed based on four dimensions of the Two-Way Social Support Scale. ${ }^{13}$ The four types of support included: (1) receiving support at the emotional level, (2) providing support at the emotional level, (3) receiving support at the instrumental level and (4) providing support at the instrumental level (online supplemental table 2).

A single item measured each support, 'If you or others required additional daily assistance, who would you depend on to assist or to be assisted by?' Receiving emotional support was conceptualised as the perception of the respondent's complaints or fears by an individual (eg, 'Do you have someone who listens to your concerns and complaints? Circle all that apply. Options included family living together, separated children and relatives, acquaintance/friends/neighbours'). Providing emotional support was conceptualised as the expression of complaints or fears by an individual to the respondents (eg, 'Do you listen to someone's concerns or complaints? Circle the numbers of all the answers that apply. Options include family living together, separated children and relatives, acquaintances/friends/ neighbours'). Receiving instrumental support was conceived as the rendering of care to the respondent by an individual, if the respondent was ill for many days (eg, 'Do you have someone who looks after you when you are sick and confined to bed for a few days? Circle the numbers of all the answers that apply. Options include family living together, separated children and relatives, acquaintance/friends/neighbours'). Providing instrumental support was defined as nursing of an individual by the respondent if they were ill for many days (eg, 'Do you look after someone when he/she is sick and confined to bed for a few days? Circle the numbers of all the answers that apply. Options include family living together, separated children and relatives, acquaintance/ friends/neighbours').

The percentage of people who responded to each item was considered while determining the level of social support. An aggregation of the responses to the survey items, apropos the four dimensions of social support, was performed for the 44 local districts and further, considered community social support indicators.

\section{Covariates}

Other explanatory variables included: gender (female, male), age (65-69, 70-74, 75-79, 80-84 and 85 years or older), living conditions (accompanied/ unaccompanied), marital status (married, never married, divorced, widowed and other/missing), education ( $\geq 13$ years, 10-12 years, $6-9$ years, $<6$ years and other/ missing), present illness (no, yes and missing), depressive symptoms evaluated by the Geriatric Depression Scale-15 (GDS-15: no depression 0-4 points, mild depression 5-9 points, depression 10-15 points, missing), smoking status (never, former, current and missing), alcohol consumption (no, do not drink every day, drink every day $\leq 35 \mathrm{~g}$ / day, drink every day $>35 \mathrm{~g} /$ day and missing) and individual social support.

\section{Statistical analysis}

This prospective study employed multilevel survival analysis. The data of 11032 people living in 44 local districts were used in this study. The multilevel analysis framework relied on the assumption that the health outcome of individuals is partially affected by the district in which they live. The multilevel model evaluated the change in outcome across districts (random effects) and the influences of community-level factors on the outcome, accounting for specific constituent features (fixed effects). Multilevel survival analysis was employed to compute the HR and 95\% CI for the onset of dementia at follow-up. The HR of the social support variable was determined as the $1 \%$ variation in the proportion of aggregated social support. For the analyses, all four social support indicators at the community level and sociodemographic factors were concurrently adjusted. Furthermore, three sensitivity analyses were conducted, excluding (1) 1 year, (2) 2 years and (3) 3years after baseline. The STATA SE V.13 (Stata Corp, College Station, Texas, USA) was used for the analysis, and the 'stmixed' command was used (the 'mestreg' command has become a standard feature in STATA V.14).

\section{Patient and public involvement}

The patients and public were not involved in the design, conduct, reporting or dissemination plans of our research.

\section{RESULTS}

During the 9.4-year follow-up period (87232 personyears), dementia onset was observed in $1776(16.1 \%)$ individuals. Online supplemental table 3 shows the baseline characteristics and incidence rate of dementia per 1000 person-years. The incidence rate of dementia was higher in those who were female, older, living alone, widowed or divorced, those having less than 6 years of education with an existing illness and with a higher score on GDS-15. It was also higher for those who did not consume alcohol, did not get support at the emotional level, did not offer support at the emotional level and did not receive help at the instrumental level, compared with each counterpart category.

Table 1 shows the mean, range, median, correlation matrix and SD of the community-level social support indicators in the 44 districts. Spearman's correlation coefficients ranged from -0.11 to 0.44 . The average proportion 
Table 1 Characteristics and Spearman's correlation coefficient matrices for community-level social support indicators ( $N=44$ school districts)

\begin{tabular}{|c|c|c|c|c|c|c|c|}
\hline & \multirow[b]{2}{*}{ Mean } & \multirow[b]{2}{*}{ SD } & \multirow[b]{2}{*}{ Minimum } & \multirow[b]{2}{*}{ Maximum } & \multicolumn{3}{|c|}{$\begin{array}{l}\text { Spearman's rank } \\
\text { correlation coefficient }\end{array}$} \\
\hline & & & & & 1 & 2 & 3 \\
\hline 2. Community-level providing emotional support & 83.1 & 2.2 & 76.1 & 88.6 & $-0.11^{*}$ & - & \\
\hline 3. Community-level receiving instrumental support & 94.0 & 1.6 & 91.3 & 97.6 & $0.44^{*}$ & $0.08^{*}$ & - \\
\hline
\end{tabular}

${ }^{*} \mathrm{P}<0.05$

of people receiving community level emotional support was $89.9 \%$, with a range of $82.7 \%-93.5 \%$. The proportion of people receiving community-level emotional support was moderately correlated with the proportion of people receiving instrumental support $(\rho=0.44)$.

The results of the multilevel survival analyses (Model 1) for the onset of incident dementia with three sensitivity analyses models (Model 2, 3 and 4) are shown in table 2 and online supplemental table 4 .

Regarding community-level social support, in Model 1, a significant association was observed between the onset of incident dementia and the proportion of people receiving community-level emotional support $(\mathrm{HR}=0.96$; $95 \% \mathrm{CI}=0.94$ to 0.99$)$. On the contrary, significant correlations or relationship between the onset of incident dementia and other community-level social support were absent. In Model 2 of a sensitivity analysis (excluding 1 year after baseline), significant correlations between the onset of incident dementia and receiving communitylevel emotional support $(\mathrm{HR}=0.97 ; 95 \% \mathrm{CI}=0.94$ to 0.99$)$ remained. Model 3 (excluding 2 years after baseline) and Model 4 (excluding 3 years after baseline) showed similar results to Models 1 and 2 (online supplemental table 4). Regarding individual-level social support, in Model 1, the incidence of dementia was significantly associated with receiving individual-level emotional support $(\mathrm{HR}=0.83$; $95 \% \mathrm{CI}=0.73$ to 0.94 ) as well as providing individual-level instrumental support $(\mathrm{HR}=0.76 ; 95 \% \mathrm{CI}=0.66$ to 0.89$)$.

\section{DISCUSSION}

To our knowledge, this is the first study to evaluate community-level social support using multilevel survival analysis to investigate the onset of dementia in a large sample of older community-dwelling individuals. There was a prospective association between living in a community with a higher level of social support and a lower occurrence of dementia during the 10-year study period. However, only one of the community-level social support indicators was significantly associated with dementia onset. The outcome of this research may have significant implications for public health, that is, by suggesting potential practical implications useful for policymakers, family members and medical staff. Because previous intervention research indicated that promoting community through salon activity increased social support in the community, ${ }^{22}$ providing such activities may be a practical

Table 2 Results of multilevel survival analyses for onset of incident dementia

\begin{tabular}{|c|c|c|}
\hline \multirow[b]{3}{*}{ Fixed effect } & Model 1 & Model 2 (1 year) \\
\hline & $n=11032$ & $n=10780$ \\
\hline & HR (95\% Cl) & HR $(95 \% \mathrm{Cl})$ \\
\hline Rate of receiving emotional support* & 0.96 (0.94 to 0.99$)$ & 0.97 (0.94 to 0.99$)$ \\
\hline Rate of providing emotional support* & 0.99 (0.96 to 1.01$)$ & 0.99 (0.96 to 1.01$)$ \\
\hline Rate of providing instrumental support* & 1.00 (0.97 to 1.03$)$ & 1.00 (0.97 to 1.02$)$ \\
\hline \multicolumn{3}{|l|}{ Random effects } \\
\hline Community-level variance (SE) & $0.06(0.05)$ & $0.04(0.06)$ \\
\hline
\end{tabular}

HR adjusted for sex, age, living alone, marital status, education, present illness, GDS, smoking status, alcohol consumption, receiving emotional support, providing emotional support, receiving instrumental support and providing instrumental support. (The full version, including individual-level results, is shown in online supplemental table 4). ${ }^{*} \mathrm{HR}$ for one-point increment of community-level social support (range: 0-100). GDS, Geriatric Depression Scale. 
solution to prevent the onset of dementia. Among older people, a $1 \%$ rise in receiving community-level emotional support correlated with an approximately $4 \%$ decrease in the incidence of dementia, irrespective of sociodemographic factors and health circumstances.

For individual-level social support, providing social support was significantly correlated with a lower risk for dementia. A previous study indicated that providing emotional and instrumental support at the individual level might be a risk factor for the onset of depression. ${ }^{13}$ A previous study by Murata and colleagues ${ }^{23}$ examined the association between individual-level social support and dementia development in a 10-year cohort. The results showed that receipt of support from friends and neighbours was associated with a lower risk of developing dementia for both men and women. Nonetheless, people who were providing social support might be less likely to develop dementia.

In the present study, among the four kinds of community social support, only community-level emotional support affected the onset of incident dementia, even after adjustment for individual-level social support. Two reasons might contribute to this finding. First, a community where people receive high emotional support from each other might be a place where older people are less likely to feel lonely. Indeed, loneliness was found to predict dementia in a previous study. ${ }^{24}$ Second, because depression was a risk factor for developing dementia, ${ }^{25}$ abundant community-level emotional support may mitigate the risk of depression, thereby decreasing the incidence of dementia. ${ }^{26}$ Therefore, a community-level indicator of receiving emotional support may be associated with the onset of dementia.

Community social support may be an element of social capital or community-level social relationships. For this reason, several plausible pathways between receiving community-level emotional support and onset of incident dementia were found in the current study. First, community-level social support may influence people's health by shaping health-associated behaviours. This may be done through faster dissemination of health-related information or by increasing the probability of people taking up healthy standards of behaviour and moderating behaviours that have negative effects on health. Second, social support may shape health by enhancing the accessibility of local services and facilities. Social involvement of older people may be fostered by accessing services, including transportation, recreational spaces, and community hubs may foster, thus, restricting or arresting the development of dementia. Third, community social support has the potential to foster good cognitive health by minimising psychological distress. Fourth, places with higher social support at the community level generate greater egalitarian political involvement trends. This may lead to the execution of policies that ensure the safety of community members. In addition, according to a systematic review of social capital including studies mainly conducted in western countries, most of the intervention studies in the last two decades have focused on individual-level changes, with a dearth of studies examining community-level changes. ${ }^{27}$ Furthermore, there are few longitudinal studies, even observational studies, that have produced dementia outcomes. Therefore, the results of this study can contribute to the social capital research agenda for developing intervention research at the community level.

It is critical to mention the possible limitations associated with the present study. First, the response rate to the survey $(52.1 \%)$ could affect the generalisability of the results. However, this response rate was higher than the census conducted by the government $(41.8 \%$ response rate in the postal survey 2020). ${ }^{28}$ Second, the dementia outcome is a nationally standardised scale used by public long-term care insurance, but it is not a clinical diagnosis. Third, there was no information about the type of dementia diagnoses (for instance, Alzheimer's disease, cerebrovascular dementia, or dementia with Lewy bodies). Fourth, the sample did not fully reflect the older population in Japan because the study subjects were recruited from a single prefecture. Therefore, the findings cannot be generalised to urban areas or places where the population has distinct characteristics. Finally, other community-level social relationships, including social capital, were not evaluated. However, we plan to assess a wider range of community-level factors in subsequent studies.

\section{CONCLUSIONS}

The results of this study showed that a higher level of social support at the community level is related to a lower incidence of dementia after adjusting for individual-level social support among older individuals. A community-level social support indicator (an aggregated value of receiving emotional support) showed a significant association with dementia onset. The present prospective study suggests that receiving emotional support at the community level may result in a lower level of incident dementia among communitydwelling older individuals in Japan.

\section{Author affiliations \\ ${ }^{1}$ Department of Social Welfare, Nihon Fukushi University, Aichi, Japan ${ }^{2}$ Department of Gerontological Evaluation, Center for Gerontology and Social Science, National Center for Geriatrics and Gerontology, Obu, Japan \\ ${ }^{3}$ Cancer Control Center, Osaka International Cancer Institute, Osaka, Japan ${ }^{4}$ Department of Oral Health Promotion, Graduate School of Medical and Dental Sciences, Tokyo Medical and Dental University, Tokyo, Japan \\ ${ }^{5}$ Center for Well-being and Society, Nihon Fukushi University, Aichi, Japan \\ ${ }^{6}$ Faculty of Health and Sport Sciences, University of Tsukuba, Tsukuba, Japan ${ }^{7}$ Department of International Health and Collaboration, National Institute of Public Health, Wako, Japan \\ ${ }^{8}$ Center for Preventive Medical Sciences, Chiba University, Chiba, Japan}

Acknowledgements Editorial support in the form of medical writing was obtained from Editage.

Contributors YM conceived the research idea, participated in the study design, performed statistical analysis and prepared the manuscript as the primary author. 
TTa developed the research idea, participated in the study design, cooperated in the statistical analysis and revised the manuscript. JA and MS assisted in the data analysis and reviewed the manuscript. TTs and YS acquired data, collaborated in statistical analysis and revised the manuscript. As the lead researcher of the JAGES project, KK helped to conceptualise the study. The final manuscript was read and approved by all authors.

Funding This study used data from the Japan Gerontological Evaluation Study (JAGES), which was supported by the Ministry of Education, Culture, Sports, Science and Technology, Japan (MEXT), Supported Program for the Strategic Research Foundation at Private Universities (grant number 2009-2013), Japan Society for the Promotion of Science (JSPS) KAKENHI (grant numbers JP18390200, JP22330172, JP22390400, JP23243070, JP23590786, JP23790710, JP24390469, JP24530698, JP24683018, JP25253052, JP25870573, JP25870881, JP26285138, JP26882010, JP15H01972, and 17K15822), Health Labour Sciences Research Grants (grant numbers H22-Choju-Shitei-008, H24-Junkanki [Seishu] Ippan-007, H24-Chikyukibo-Ippan-009, H24-Choju-Wakate-009, H25-Kenki-Wakate-015, H25-Choju-Ippan-003, H26-Irryo-Shitei-003 [Fukkou], H26-Choju-Ippan-006, H27-Ninchisyou-Ippan-001, H28-choju-Ippan-002, H28Ninchisyou-Ippan-002, H30-Kenki-Ippan-006 and H30-Junkankitou-Ippan-004), Japan Agency for Medical Research and development (AMED) (grant numbers JP17dk0110017, JP18dk0110027, JP18Is0110002 and JP18le0110009) and the Research Funding for Longevity Sciences from National Center for Geriatrics and Gerontology (grant numbers 24-17, 24-23, 29-42 and 30-22).

Disclaimer The views and opinions expressed in this article are those of the authors and do not necessarily reflect the official policy or position of the respective funding organisations.

Competing interests None declared.

Patient consent for publication Not required.

Ethics approval The ethics committee of Research of Human Subjects at the Nihon Fukushi University evaluated and approved the use of the JAGES protocol (approval number 13-14). Consent to participate in the study was indicated by a written explanation at the beginning of the questionnaire and by the response received on the questionnaire.

Provenance and peer review Not commissioned; externally peer reviewed.

Data availability statement Data are available upon reasonable request.

Supplemental material This content has been supplied by the author(s). It has not been vetted by BMJ Publishing Group Limited (BMJ) and may not have been peer-reviewed. Any opinions or recommendations discussed are solely those of the author(s) and are not endorsed by BMJ. BMJ disclaims all liability and responsibility arising from any reliance placed on the content. Where the content includes any translated material, BMJ does not warrant the accuracy and reliability of the translations (including but not limited to local regulations, clinical guidelines, terminology, drug names and drug dosages), and is not responsible for any error and/or omissions arising from translation and adaptation or otherwise.

Open access This is an open access article distributed in accordance with the Creative Commons Attribution Non Commercial (CC BY-NC 4.0) license, which permits others to distribute, remix, adapt, build upon this work non-commercially, and license their derivative works on different terms, provided the original work is properly cited, appropriate credit is given, any changes made indicated, and the use is non-commercial. See: http://creativecommons.org/licenses/by-nc/4.0/.

\section{ORCID iDs}

Yasuhiro Miyaguni http://orcid.org/0000-0002-4973-1192

Takahiro Tabuchi http://orcid.org/0000-0002-1050-3125

Jun Aida http://orcid.org/0000-0002-8405-9872

Masashige Saito http://orcid.org/0000-0002-3997-3884

Taishi Tsuji http://orcid.org/0000-0002-8408-6619

Katsunori Kondo http://orcid.org/0000-0003-0076-816X

\section{REFERENCES}

1 Prince M, Wimo A, Guerchet M. World Alzheimer report 2015: the global impact of Dementia: an analysis of prevalence, incidence, cost and trends [online report]. London: Alzheimer's Disease International, 2015. https://www.alz.co.uk/research/WorldAlzheimerReport2015. pdf

2 Ministry of Health Law. 認知症施策推進大綱, 2017. Available: https:// www.mhlw.go.jp/content/000522832.pdf
3 Middleton LE, Yaffe K. Promising strategies for the prevention of dementia. Arch Neurol 2009:66:1210-5.

4 Fratiglioni L, Paillard-Borg S, Winblad B. An active and socially integrated lifestyle in late life might protect against dementia. Lancet Neurol 2004;3:343-53.

5 Gao S, Hendrie HC, Hall KS, et al. The relationships between age, sex, and the incidence of dementia and Alzheimer disease: a metaanalysis. Arch Gen Psychiatry 1998;55:809-15.

6 Livingston G, Sommerlad A, Orgeta V, et al. Dementia prevention, intervention, and care. Lancet 2017;390:2673-734.

7 Norton MC, Dew J, Smith H, et al. Lifestyle behavior pattern is associated with different levels of risk for incident dementia and Alzheimer's disease: the Cache County study. J Am Geriatr Soc 2012;60:405-12.

8 Patterson C, Feightner JW, Garcia A, et al. Diagnosis and treatment of dementia: 1. risk assessment and primary prevention of Alzheimer disease. CMAJ 2008;178:548-56.

9 Santini ZI, Koyanagi A, Tyrovolas S, et al. The association between social relationships and depression: a systematic review. J Affect Disord 2015;175:53-65.

10 Heany CA, Israel BA. Social networks and social support. In: Glanz $\mathrm{K}$, Rimer BK, Viswanath K, eds. Health behavior and health education theory, research, and practice. 4th ed. San Francisco, CA: JosseyBass, 2008: 189-210.

11 Seeman TE, Lusignolo TM, Albert M, et al. Social relationships, social support, and patterns of cognitive aging in healthy, high-functioning older adults: MacArthur studies of successful aging. Health Psychol 2001;20:243-55.

12 Holt-Lunstad J, Smith TB, Layton JB. Social relationships and mortality risk: a meta-analytic review. PLoS Med 2010;7:e1000316.

13 Tsuboi H, Hirai H, Kondo K. Giving social support to outside family may be a desirable buffer against depressive symptoms in community-dwelling older adults: Japan gerontological evaluation study. Biopsychosoc Med 2016;10:18.

14 Brown SL, Nesse RM, Vinokur AD, et al. Providing social support may be more beneficial than receiving it: results from a prospective study of mortality. Psychol Sci 2003;14:320-7.

15 Saito T, Murata C, Saito M, et al. Influence of social relationship domains and their combinations on incident dementia: a prospective cohort study. J Epidemiol Community Health 2018;72:7-12.

16 Berkman LF, Kawachi I. Social epidemiology. New York, NY: Oxford University Press, 2015.

17 Aida J, Kondo K, Kawachi I, et al. Does social capital affect the incidence of functional disability in older Japanese? A prospective population-based cohort study. J Epidemiol Community Health 2013;67:42-7.

18 Murayama $\mathrm{H}$, Sugiyama $\mathrm{M}$, Inagaki $\mathrm{H}$, et al. Is community social capital associated with subjective symptoms of dementia among older people? A cross-sectional study in Japan. Geriatr Gerontol Int 2018;18:1537-42.

19 Nishi A, Kondo K, Hirai H, et al. Cohort profile: the ages 2003 cohort study in Aichi, Japan. J Epidemiol 2011;21:151-7.

20 Hisano S. The relationship between revised Hasegawa dementia scale (HDS-R), Mini-Mental state examination (MMSE) and Bed-fast scale, dementia scale. Nihon Ronen Igakkai Zasshi 2009;20:883-91.

21 Takagi D, Kondo K, Kawachi I. Social participation and mental health: moderating effects of gender, social role and rurality. BMC Public Health 2013;13:701.

22 Murayama H, Kondo K, Fujiwara Y. Global perspectives on social capital and health [original in Japanese]. In: Kawachi I, Takao S, Subramanian SV, eds. Social capital to Kenkou Seisaku. 1st ed. Tokyo: Nihon Hyoronsha, 2008: 189-210.

23 Murata C, Saito T, Saito M, et al. The association between social support and incident dementia: a 10-year follow-up study in Japan. Int J Environ Res Public Health 2019;16:239.

24 Holwerda TJ, Deeg DJH, Beekman ATF, et al. Feelings of loneliness, but not social isolation, predict dementia onset: results from the Amsterdam study of the elderly (AMSTEL). $J$ Neurol Neurosurg Psychiatry 2014:85:135-42.

25 Ownby RL, Crocco E, Acevedo A, et al. Depression and risk for Alzheimer disease: systematic review, meta-analysis, and metaregression analysis. Arch Gen Psychiatry 2006;63:530-8

26 Saito M, Kondo N, Aida J, et al. Development of an instrument for community-level health related social capital among Japanese older people: the JAGES project. J Epidemiol 2017;27:221-7.

27 Villalonga-Olives E, Wind TR, Kawachi I. Social capital interventions in public health: a systematic review. Soc Sci Med 2018;212:203-18.

28 Ministry of Health, Labor and Welfare. Announcement of the end of the survey period for the 2020 National Census [original in Japanese], 2020. Available: https://www.stat.go.jp/data/kokusei/ 2020/houdou/pdf/20201021.pdf 